\title{
Modelo para Fortalecer Capacidades Básicas de Pyme
}

\author{
Bernardo Pérez Castaño*
}

* Ph.D. Universidad Politécnica de Valencia - España. Profesor Titular de la Escuela de Ingeniería Industrial y Estadística de la Universidad del Valle - Santiago de Cali, Colombia.

E-mail:beperez@univalle.edu.co
Fecha de recepción: Junio 22 de 2003

Fecha de aprobación: Julio 2 de 2003

\section{RESUMEN}

En este artículo se presentan los aspectos clave de un modelo para fortalecer las capacidades básicas de pyme manufactureras, siendo su eje central un conjunto de estrategias de competitividad basadas en la gestión del conocimiento en el marco de un Sistema de Conocimiento denominado DICES: DATOS, IMFORMACIÓM, COMOCIMIEMTO, EMTEMDIMIENTO, SABIDURÍA. EI modelo pretende encauzar a los empresarios hacia una gestión estratégica basada en metodologías caracterizadas por: tener un sentido más racional que empírico; utilizar procedimientos sistemáticos; ser sencillas, lógicas y de sentido 
común; fáciles de asimilar y de poner en práctica en las pyme; considerar el pragmatismo de los empresarios sin desconocer la rigurosidad científica; concentrarse en lo vital (procesos, conocimientos y capacidades centrales del negocio); tener un enfoque holístico, respetando la inmediatez característica de los empresarios, sin dejar de lado la importancia estratégica del largo plazo; además contribuyen al desarrollo del pensamiento estratégico de los empresarios y a la formación, tanto de capital social como de una sociedad más culta, representada en el personal formado y capacitado. Parte esencial del modelo lo constituye el fortalecimiento de la capacidad de asimilar información estratégica en las pyme y para ello se recomienda dotar al personal de las capacidades DIA: Desaprender, Innovar, Aprender.

PALABRAS ClAVE: Capacidades básicas empresariales, estrategia, competitividad, gestión del conocimiento, pyme

\section{ABSTRACT}

In this paper the key aspects of a model are presented to strengthen the basic capacities of pyme manufacturers, being their central axis a group of strategies of competitiveness based on the knowledge management in the mark of a Knowledge System denominated DIKUW: Data, Information, Knowledge, Understanding, Wisdom. The pattern seeks to channel the managers toward a strategic administration based on methodologies characterized for: to make a more rational sense that empiric; to use systematic procedures; to be simple, logical and of common sense; easy to assimilate and of putting into practice in the pyme; to consider the pragmatism of the managers without ignoring the scientific method; to concentrate on the vital thing (processes, knowledge and central capacities of the business); to have a holistic focus, respecting the short term characteristic of the managers, without leaving aside the strategic importance of the long term; they also contribute to the development of the strategic thought of the managers and the formation, so much of social capital as of a more learned society, represented in the formed personnel and qualified. It leaves essential of the pattern it constitutes it the invigoration of the capacity of assimilating strategic information in the pyme and for it is recommended it to endow the personnel of the capacities FIL: Forget, Innovate, Learn.

KEY WORDS: Managerial basic capacities, strategy, competitiveness, knowledge management, pyme

\section{INTRODUCCIÓN}

La empresa se ha constituido como la institución social y económica más poderosa de la sociedad moderna. Versátil y creativa, la empresa es un prodigioso amplificador del esfuerzo humano más allá de las fronteras nacionales y culturales. Las empresas, y no las fuerzas económicas abstractas o los gobiernos, son las que crean y distribuyen la mayor parte de una economía, la innovación y el comercio, elevando así el nivel de vida [1][2][3]. En casi todos los países desarrollados o en vías de desarrollo, la pyme tiene un papel protagonista en la generación de empleo y en el crecimiento económico, a la vez que constituyen un elemento de cohesión territorial y social de primera magnitud [4][5]. La internacionalización ha llevado la competencia más allá de las multinacionales; las pyme también se ven afectadas por los nuevos retos del mundo empresarial [6]. Concretamente en Colombia, las transformaciones de su economía desde inicios de los años noventa han definido nuevas condiciones para el funcionamiento de las empresas; la apertura comercial y la desregulación económica han generado un marco que plantea nuevas demandas de competitividad para las pyme.

En esta investigación se abordó como problema la competitividad de la pyme manufacturera del Valle del Cauca (Colombia), partiendo de la hipótesis que, en general, no tienen procesos ni 
actividades, estándares y sistemáticas, asociadas con la gestión del conocimiento como una estrategia básica para desarrollar capacidades que faciliten la formulación e implantación exitosa de sus estrategias tanto funcionales, como de negocios y corporativa. Se investigaron 66 pyme de cinco agrupaciones industriales, tanto para conocer como para entender su estado actual y obtener elementos clave con base en los cuales se formularon las estrategias de competitividad. La encuesta se diseñó con base en el marco conceptual y en la experiencia tanto investigadora como profesional. El conocimiento obtenido mediante la encuesta fue profundizado, complementado y confrontado con los empresarios de las pyme encuestadas, para lograr mayor entendimiento de la problemática y de las características de las empresas y su gestión. Las ideas y propuestas formadas posteriormente fueron contrastadas mediante entrevistas profundas y detalladas en 5 pyme de Valencia (España).

\section{ASPECTOS CLAVE DE LA GESTIÓN DEL CONOCIMIENTO EN LA PYME}

\section{Generalidades sobre la pyme colombiana:}

Diversos estudios concluyen que la débil competitividad de Colombia se debe en buena parte a la fragilidad de su microeconomía empresarial. Hasta 1990, la pyme dependía más de su habilidad en materia de gestión financiera de corto plazo que de su gestión estratégica global. Actualmente muy pocas pyme están orientadas hacia mercados externos en forma estratégica. Además, la elevada inestabilidad socioeconómica del país atenta contra el incipiente proceso exportador.

\section{Hechos significativos observados en la gestión de la pyme vallecaucana:}

$\square$ En general, la base de recursos y capacidades con que cuentan es poco adecuada para competir en las actuales condiciones del mercado. Sin embargo, sus empresarios poseen características muy positivas como madurez, pericia, experiencia, formación profesional y fuerte orientación hacia la acción que podrían ser mejor aprovechadas.

$\checkmark$ En general, adoptan parcialmente, técnicas y metodologías de gestión buscando resultados inmediatos, lo que conduce a atacar los síntomas de los problemas, sin analizar ni tomar acciones sobre las causas que los originan. Mo crean el ambiente propicio para introducir los cambios necesarios que conduzcan a una nueva cultura empresarial, con una filosofía, valores y principios como soporte de dichas técnicas.

$\checkmark$ Los productos y procesos novedosos no constituyen una fortaleza ni una debilidad importante para los empresarios. La imagen ideal de los productos de la empresa es poco conocida por su personal.

$\square$ Se percibe poco interés en los empresarios por identificar mercados potenciales y conocer sus características. Los vínculos de la pyme con su entorno son escasos, sus relaciones con otras instituciones, inspiradas en un objetivo común, son poco frecuentes.

$\square$ En general, los empresarios de la pyme tienen dificultad para identificar fuentes y contenidos relevantes de información tecnológica. estudio refleja dificultades en la obtención y tratamiento de los conocimientos esenciales para tomar decisiones racionales. En la compra de máquinas no se considera relevante analizar los aspectos intangibles de la tecnología.

च En general, es poca la importancia que se le da a la adquisición y desarrollo de las capacidades tecnológicas del personal. La capacitación carece de enfoque integral, sin una relación evidente con la estrategia u objetivos de la empresa y responde a aspectos puntuales de la actuación más que a planes de desarrollo. Mo sólo hay falta de capacitación sino de identificación de necesidades de capacitación. Las habilidades adquiridas por el personal en la empresa son más una consecuencia del aprendizaje a través del error-ensayo, que el resultado de programas intencionados de 
capacitación. Mo se nota interés por garantizar la asimilación del saber externo por parte de los trabajadores de la organización e integrarlo al saber corporativo. Mo se aprovecha a fondo la experiencia y la iniciativa de las personas que trabajan en ella para la búsqueda y análisis de posibles soluciones a los problemas cotidianos en el área operativa. Parece que los empresarios no se percatan que los procesos deben ser registrados para formalizar el saber técnico y evitar riesgos de que el know how empresarial se vaya con las personas cuando se retiren de la organización.

$\checkmark$ Hay deficiencias en la planificación, programación y evaluación participativa de los trabajadores y en la comunicación. En general, los componentes de la gestión del talento humano (selección, inducción, entrenamiento, capacitación, evaluación) están muy poco desarrollados dentro de las empresas; los directivos no integran la gestión humana como parte de sus funciones gerenciales.

\section{Hechos significativos observados en la gestión de la pyme valenciana:}

Poseen estrategia competitiva para sobrevivir, planes de expansión basados en la ampliación de cartera de productos, con prioridad en diseño, calidad en la fabricación y servicio al cliente. Realizan esfuerzos en mercadeo, desarrollo tanto de productos como de procesos y de capital humano. Han establecido vínculos con entidades externas y consolidado clusters.

\section{MODELO PARA FORTALECER CAPACIDADES BÁSICAS DE PYME}

El eje central del modelo propuesto está conformado por un sistema de conocimiento aunado a un sistema de capacidades, cuya implementación se soporta en estrategias basadas en la gestión del conocimiento orientadas hacia la adquisición y el fortalecimiento de la capacidad de asimilar información estratégica, la capacidad estratégica, la capacidad tecnológica y la capacidad de innovación, en esencia la capacidad de actuación de la pyme.

El modelo está enmarcado en el contexto de la competitividad y de la gestión empresarial actual, donde se presentan interacciones entre los cambios que ocurren en el mercado y en el orden económico internacional, los cuales van definiendo las tendencias en el paradigma competitivo, dando paso a diferentes énfasis en el paradigma de gestión, cuyas directrices recomiendan administrar de manera eficiente $y$ efectiva los procesos mediante los cuales se relacionan las diversas funciones; con un enfoque, sobre todo, en la capacidad de análisis estratégico profundo y en la capacidad para olvidarse de habilidades viejas y aprender lo nuevo [11] [12], en atención a la importancia creciente de la creación y producción de información y conocimiento para el desarrollo social y económico, característica del nuevo contexto económico. A su vez, se abren nuevas perspectivas de inserción competitiva para la pyme, aunque simultáneamente ven surgir competidores inesperados, productos y procesos sustitutos, cambios en las preferencias de clientes y consumidores [9]. Como respuesta, la pyme debe echar mano de sus recursos y capacidades para crear estrategias (corporativa, de negocios y funcionales) y a su vez diseñar estrategias internas con el fin de adquirir nuevos recursos y capacidades.

La propuesta es utilizar la gestión del conocimiento como estrategia interna para adquirir competencias que se transformen en capacidades, a través de las cuales se puedan adquirir más conocimientos que conduzcan al desarrollo de nuevas capacidades, generándose así un círculo virtuoso.

\section{Sistema de Conocimiento DICES:}

La gestión del conocimiento como estrategia básica per se no es suficiente, se requiere implementarla en un sistema de conocimiento 
que permita consolidar el conocimiento corporativo de la pyme, en el contexto de los procesos del negocio, a partir del conocimiento individual de su personal apoyado por la cultura organizacional y los medios tecnológicos con que cuentan para apoyar dicha gestión. Se propone para ello el sistema de conocimiento DICES, el cual indica la construcción progresiva de valor agregado en el ciclo conformado por Datos, Información, Conocimiento, Entendimiento y Sabiduría. DICES además de ser el acrónimo de los elementos mencionados destaca la importancia de la comunicación que debe existir en la pyme, en todos los sentidos, niveles y temas de la organización.

Elementos del Sistema de Conocimiento DICES:

Los datos, entendidos como cifras o hechos, se convierten en información relevante cuando son relacionados de manera creativa; para ello deben ser ordenados o agrupados, siendo éste el primer proceso de incorporación de valor agregado.

\section{La información se transmite mediante descripciones, respuestas a preguntas tales como quién, qué, cuándo, dónde y cuántos}

El conocimiento surge de la interpretación y reflexión de datos e información realizada en un contexto determinado, sus implicaciones se hacen explícitas en un contexto [13] [14] por expertos, o sea con base en el saber y la experiencia propia de las personas, conformándose entonces el segundo proceso de incorporación de valor agregado para una organización específica [15]. De esta manera el conocimiento adquiere un valor de uso con posibilidades de ser convertido en un valor de cambio representado en la tecnología, es decir mediante la aplicación práctica del conocimiento, que constituye un saber hacer, el cual puede ser patentado y vendido. Por lo tanto, se reúnen datos para producir información y generar conocimiento. Surgiendo aquí una importante premisa, aunque aparentemente muy sencilla: de datos equivocados no es posible extraer información correcta. Cumplir esta premisa garantiza que el conocimiento obtenido permita saber qué es correcto y además cómo se hace. La vía más usual para obtener y transmitir el conocimiento es a través del pensamiento analítico.

\section{El conocimiento se transmite mediante instrucciones, respuestas a preguntas sobre cómo}

El entendimiento implica saber por qué es correcto y por qué se hace, a partir de saber qué es correcto y además de cómo se hace, esto es a partir del conocimiento obtenido. La vía más usual para obtener y transmitir el entendimiento es a través del pensamiento sintético.

\section{El conocimiento se transmite mediante explicaciones, respuestas a preguntas como por qué}

Finalmente, la sabiduría tiene que ver con valores, implica el ejercicio del juicio. Sabiduría es la capacidad de incrementar la eficacia, mientras que inteligencia es la capacidad de incrementar la eficiencia [14].

Adquirir y generar conocimiento puede ser suficiente para que una empresa sobreviva, pero no lo es para que se destaque y sobresalga por encima de la mayoría, ni mucho menos para que sea líder en algún aspecto en su sector. Para sobresalir se requiere generar entendimiento y para ser líder, desarrollar sabiduría. Por lo tanto, la gestión del conocimiento debe estar orientada hacia el entendimiento, para que la empresa mejore su posición competitiva y ascienda de nivel, de sobrevivir a sobresalir y posteriormente a la cúspide, ser líder. El conocimiento le permite a la empresa mantenerse en el primer nivel. Mientras que, mediante la gestión del conocimiento la empresa puede avanzar hacia el entendimiento, mejorar la eficiencia e implantar estrategias para ascender al siguiente nivel. 
Finalmente, para llegar a la cima se requiere mejorar la eficacia y desarrollar una ventaja competitiva que distinga a la empresa por superar a las demás en algún aspecto. Para obtener y mantener una ventaja competitiva se precisa lograr la sabiduría mediante la aplicación de juicios y valores al entendimiento. Esto último implica desarrollar la gestión del conocimiento con una visión estratégica y considerarla inmersa en el Sistema de Conocimiento DICES.

\section{GESTIÓN DEL CONOCIMIENTO PARA EL ENTENDIMIENTO}

\begin{abstract}
El Análisis se enfoca en la estructura; revela cómo funcionan los objetos, mira a su interior, reduce el centro de atención.

En el Pensamiento Analítico el objeto por explicar se trata como un todo que debe separarse; sirve para la obtención y transmisión de conocimiento.

El conocimiento implica saber cómo se hace y qué es correcto, permite describir.
\end{abstract}
La Sintesis se enfoca en la función; revela porqué los objetos operan como lo hacen, mira a su exterior, expande el centro de atención.
En el Pensamiento Sintético el objeto por explicar se trata como una de las partes del todo contenedor, sirve para la obtención y transmisión de entendimiento. El entendimiento implica saber por qué se hace y por qué es correcto, permite explicar.

El Pensamiento Sistémico combina los dos, su tarea fundamental es desarrollar la complementariedad del pensamiento analítico y sintético: lograr que las partes identificadas por el análisis de las funciones que debe llevar a cabo el todo, encajen entre sí a fin de trabajar juntas armoniosamente, así como con eficiencia y eficacia.

El crecimiento en la cantidad de información disponible aumenta la demanda en las habilidades para seleccionar y usar la información inteligentemente. Por ello, tanto la información como la tecnología de la información deben estar integradas en la cartera de instrumentos competitivos para mejorar los resultados empresariales [16]. La misma tecnología, especialmente los medios de comunicación electrónicos, contribuye a intensificar el proceso de aceleración de los flujos de tecnología por todo el mundo [17]. La solución de muchos aspectos productivos está en la capacidad de integrar corrientes tecnológicas y de coordinar las diversas técnicas de producción como un resultado del aprendizaje colectivo de la organización [18] [19].

De otra parte, la innovación como proceso a través del cual las ideas llegan al mercado en forma de nuevos o mejorados productos y/o servicios, está compuesto por dos partes no necesariamente secuenciales y con frecuentes caminos de ida y vuelta entre ellas. Una de las partes se ocupa de la creación de conocimiento, mientras que la otra de la aplicación del conocimiento creado para convertirlo en un proceso, un producto o un servicio que incorpore nuevas características satisfactorias para el mercado y que representen ventajas competitivas para la empresa [20][21][22][23]. Por ello, para culminar con éxito el proceso innovador la empresa debe: involucrar la innovación en su estrategia; tener una organización apropiada para innovar; dominar los conocimientos tecnológicos en que se fundamenta su innovación [24][25][26][27]. Por lo tanto, se requiere tener un clima empresarial 
propicio para convencer y motivar al personal de crear, revelar, compartir y utilizar el conocimiento. Para ello es preciso considerar que la organización es inteligente de manera similar que los seres humanos. La inteligencia de una organización es una función de la organización de la inteligencia individual de las personas dispersas en ella y de las conexiones entre éstas y los sistemas de los cuales ellas forman parte; siendo factor crucial, la capacidad cognoscitiva del sistema completo [28][29][30][31][32]. De ahí que la empresa deba conocer no sólo el rendimiento de sus empleados en sus tareas actuales y pasadas sino también el repertorio de sus destrezas y habilidades [33]. Pero, la capacidad de la organización depende no sólo de sus recursos sino también de la habilidad para integrarlos, la cual a su vez está ligada a la capacidad de relación entre los empleados, que a su vez es un resultado de otro recurso intangible: la cultura de la organización [34]. Por lo tanto, el personal con diferentes niveles de especialización tiene entre sus manos la clave de la tecnología; ellos manejan los materiales, controlan los equipos, toman parte de las decisiones, administran los demás recursos productivos y acumulan gran parte de la experiencia de la empresa [35][36][37][38]. Los principales recursos corporativos están constituidos por el conocimiento y experiencia del personal de primera línea con su iniciativa, creatividad y diversidad, valores clave en el actual paradigma competitivo [39].
Finalmente, tenemos la gestión del conocimiento involucrando dos aspectos relevantes. Por una parte la idea de gestión indica, la organización, la planificación, la dirección y el control de procesos para conformar o disponer de ciertos objetivos [40]. De otro lado, al hablar de conocimiento se pone de manifiesto que una organización, como cualquier ser humano, está sometida a una dinámica en la que del exterior y del interior mismo, capta o percibe información, la reconoce, la organiza, la almacena, la analiza, la evalúa y emite una respuesta al exterior, basada en dicha información y englobada en el total de Información almacenada, procurando un resultado [41][42][43][44][45][46][47][48]. Frente a la dinámica expuesta, la organización puede desarrollar estrategias basadas en la gestión del conocimiento, mediante la dotación de competencias de conocimiento a su personal, de tal manera que, a su vez, adquiera capacidad de actuación, para mejorar su posición competitiva.

\section{ESTRATEGIA PARA ADQUIRIR CAPACIDAD DE ASIMILAR INFORMACIÓN ESTRATÉGICA}

Lo crucial en la pyme del Valle del Cauca está en adquirir la capacidad de asimilar información estratégica, para lo cual se requiere que al interior desarrollen prácticas fundamentales de gestión y, al exterior desarrollen un fuerte entramado de colaboración con otras empresas e instituciones.

\begin{tabular}{|l|l|}
\hline \multicolumn{1}{|c|}{ ESTRATEGIA IMTERMA } & \multicolumn{1}{c|}{ ESTRATEGIA EXTERMA } \\
\hline $\begin{array}{l}\text { Prácticas fundamentales de gestión } \\
\begin{array}{l}\text { Identificación de conocimientos } \\
\text { pertinentes, relevantes y esenciales }\end{array}\end{array}$ & $\begin{array}{l}\text { Fuertes nexos de colaboración } \\
\square \text { Desarrollo de vínculos con otras empresas } \\
\text { e instituciones } \\
\begin{array}{l}\text { Adquisición de información } \\
\text { Facilitar el proceso a más información } \\
\text { Transferencia de información }\end{array}\end{array}$ \\
\end{tabular}


La sinergia del desarrollo de las prácticas de gestión y del entramado de colaboración, produce como resultado un efecto de círculo virtuoso en pro del incremento de la capacidad de asimilación de información estratégica, convirtiendo esta situación en una espiral ascendente de fortalecimiento mutuo, de tal manera que la capacidad de asimilación de información estratégica adquirida le permite a la empresa identificar y adquirir más información estratégica, reforzando dicha capacidad y, así sucesivamente. A su vez, la información estratégica adquirida debe ser transformada en conocimiento, el cual, mediante su aplicación, da origen a nuevos conocimientos.

Se hace explícita la definición de gestión del conocimiento como el proceso de identificar, adquirir, utilizar y crear, tanto datos como información y conocimientos, relevantes, externos e internos a la organización, para mejorar tanto la eficiencia como la eficacia de la empresa con el fin de garantizar su permanencia en el escenario competitivo. La gestión del conocimiento vista así es un camino para adquirir e incrementar la capacidad de asimilación de información estratégica, a su vez, para mejorar la capacidad estratégica, la capacidad tecnológica y la capacidad de innovación de la pyme. Además, para consolidar el pensamiento estratégico en los empresarios e introducir procesos y procedimientos sistemáticos en la gestión de la pyme, de tal manera que se distinga por formular claramente los problemas y examinar críticamente diversas soluciones con la racionalidad y la crítica que caracteriza el método científico. Simultáneamente, aprovechar deliberadamente el saber conformado por los conocimientos y experiencias del personal, acumuladas con el paso del tiempo, facilitando así tanto la detección de los efectos de los problemas como la determinación de las causas que los originan.

Componentes del Sistema de Conocimiento DICES:

La interacción de los componentes del Sistema de Conocimiento DICES facilita la transformación y su consecuente agregación progresiva de valor a los elementos DICES, mediante un proceso continuo, sistemático y cotidiano, en el cual se logra un conocimiento corporativo orientado hacia la eficacia y la eficiencia de los procesos del negocio, superior a la suma de los conocimientos individuales de las personas de la organización, a través de la cultura organizacional y con el apoyo de los medios tecnológicos. De esta manera, la pyme puede lograr mejorar su posición competitiva, pasando de un estado de sobrevivir a sobresalir y por último a ser líder. Se trata de obtener Conocimiento Corporativo enfocado en los Procesos del Megocio a partir del conocimiento individual del Personal de la pyme, con el apoyo de la Cultura Organizacional y los Medios Tecnológicos.

El personal de la organización. Desde los inicios del siglo XX en las empresas se viene repitiendo la frase "las personas son nuestro principal recurso". Pero aún son muy pocos los directivos que realmente se preocupan de ese recurso [50]. Por excelencia, es el personal de la pyme el primero y más importante componente del Sistema de Conocimiento DICES. La funcionalidad y prosperidad de una organización depende, principalmente, del conocimiento y las habilidades de su personal, y del conocimiento corporativo que desarrollen y difundan colectivamente. Los conocimientos de las personas, fruto de sus estudios, vivencias y experiencias, aunados a su inherente racionalidad, su capacidad de análisis, de entendimiento y de asimilación, son vitales para la transformación de los elementos del Sistema y la obtención de sus beneficios, representados finalmente en el mejoramiento de la posición competitiva de la empresa.

La cultura organizacional. Puede verse como un conjunto de ideas, creaciones, creencias, costumbres, hábitos, aptitudes, valores, símbolos y mitos que caracterizan a una organización [51]. También como un sistema dinámico de rubros dependientes tales como 
ambiente, ética, visión, valores, estrategias, actitudes, comportamientos y acciones [52]. La cultura organizacional tiene un carácter descriptivo; se refiere a la manera como las personas que componen la organización toman posiciones y enfrentan el desarrollo de ésta sin evaluar ese posicionamiento. Una organización es entonces producto de las personas que la conforman. Su eficacia para alcanzar lo que se haya propuesto está influenciada de manera decisiva por la cultura que en su interior se desarrolla. Por lo tanto, en la pyme se requiere una cultura organizacional que facilite y apoye la transformación y agregación progresiva de valor a los elementos del Sistema de Conocimiento DICES, mediante un proceso continuo, sistemático y cotidiano, para lograr un conocimiento corporativo orientado hacia la eficacia y la eficiencia de los procesos del negocio, superior a la suma de los conocimientos individuales de las personas de la organización, aprovechando su enriquecimiento como producto de la sinergia entre los componentes del Sistema de Conocimiento DICES.

Los medios tecnológicos. Son herramientas que dan apoyo al proceso central de gestión del conocimiento corporativo facilitando el proceso de adición de valor a los elementos del Sistema de Conocimiento DICES. Sin embargo, los medios tecnológicos no son suficientes puesto que el conocimiento se transmite más es a través de una red humana. El Sistema de Conocimiento DICES considera la existencia de procesos organizacionales que buscan sinergia entre la capacidad de procesamiento de datos e información y la capacidad creativa e innovadora del personal de una organización. El solo hecho de disponer de las tecnologías, por buenas que sean, no es suficiente para asegurar la aplicabilidad de la información, se requiere, además, de la capacidad creativa e innovadora de las personas para lograr la competencia organizacional.

El conocimiento corporativo. Las actividades productivas en la pyme son cada vez más especializadas y requieren de personas con conocimientos más profundos sobre determinados temas. Para el análisis de los problemas que se presentan en las distintas etapas de los procesos se requiere de la participación conjunta de los especialistas, con visión global, aportando y compartiendo cada uno sus conocimientos individuales hasta llegar a la implantación de la solución seleccionada, creando así el conocimiento corporativo [53]. Este conocimiento se debe ir registrando y actualizando sistemáticamente dando origen a lo que se denomina la memoria corporativa, base de conocimiento que da soporte a la organización. En esta memoria se ve representada la sinergia producida por la cultura de la organización, mediante la cual se logra que sea más que la suma de la memoria fragmentada de las personas que trabajan en ella. El aprendizaje ocurre, así no exista una estructura formal, siempre y cuando se den las vinculaciones necesarias entre las diferentes actividades involucradas en el proceso del negocio con una retroalimentación que conecte la información correspondiente a cada una de esas actividades, reforzado con los medios tecnológicos y la existencia para que sea posible aprender y no sólo para que la organización tenga memoria [54][55]. De esta manera la pyme puede registrar los acontecimientos para aprender de sus propios errores y aciertos.

Los procesos del negocio. La pyme debe definir los objetivos de su negocio en función de sus productos y procesos, de tal manera que dicha definición actúe como un faro que oriente permanentemente su quehacer y facilite una diferenciación clara entre los procesos centrales del negocio (aquellos que estén directamente involucrados en la generación de productos y servicios para los clientes externos) y los de apoyo (los que lo estén para los clientes internos).

\section{ESTRATEGIAS PARA IMPLEMENTAR EL SISTEMA DE CONOCIMIENTO DICES}

Asociadas a cada fase de la gestión del conocimiento se proponen estrategias para 
lograr la implementación y adaptación del Sistema de Conocimiento DICES. Las estrategias se presentan de manera aislada pero cabe recordar que al tratarse de un sistema no se pueden dejar a un lado las cadenas de efectos, interdependencias e interrelaciones que se dan, tanto entre dichas estrategias como entre las fases de la gestión del conocimiento.

\begin{tabular}{|c|c|c|}
\hline \multicolumn{2}{|c|}{ Estrategias para la Implementación del Sistema de Conocimiento DICES } \\
\hline $\begin{array}{c}\text { FASE GESTIÓM } \\
\text { DEL COMOCIMIEMTO }\end{array}$ & ESTRATEGIAS IMTERMAS & ESTRATEGIAS EXTERMAS \\
\hline Identificación & $\begin{array}{c}\text { Clasificación del Conocimiento } \\
\text { en pertinente, relevante y } \\
\text { esencial }\end{array}$ & $\begin{array}{c}\text { Vigilancia Tecnológica y del } \\
\text { paradigma competitivo }\end{array}$ \\
\hline Adquisición & $\begin{array}{c}\text { Redes internas, tanto formales } \\
\text { como informales }\end{array}$ & $\begin{array}{c}\text { Redes externas, asesorías, } \\
\text { alianzas, fusiones, patentes, } \\
\text { franquicias }\end{array}$ \\
\hline Utilización & Competencias laborales \\
Creapacidades DIA & $\begin{array}{c}\text { Institutos y Centros de } \\
\text { capacitación empresariales }\end{array}$ \\
\hline Creación & Condiciones para la innovación & I+D+I+C \\
\hline
\end{tabular}

I+D $+I+C=$ Investigación y Desarrollo para la Innovación en ambientes de Cooperación

\section{Competencias laborales y capacidades DIA para el entendimiento.}

Está visto que sí la gestión del conocimiento se orienta para que el personal de la empresa entienda sus procesos, funciones y tareas, se obtendrá el conocimiento corporativo que facilitará el camino para mejorar su posición competitiva. Se plantea entonces la dotación de competencias laborales a todos los trabajadores de las pyme como la vía para conseguir este entendimiento. Para establecer una comunicación eficaz se debe hablar la misma jerga, por lo tanto todos los miembros de la empresa deben tener los conocimientos básicos sobre el producto, el proceso y el mercado.

La dotación de competencias laborales está estrechamente asociada con la adquisición de Capacidades DIA: Desaprender, Innovar y Aprender. Capacidad de desaprender, para abrir la mente a la creatividad; capacidad de innovar, para orientar la creatividad hacia resultados concretos y favorables para la empresa; capacidad de aprender, para discernir qué es lo relevante, pertinente y esencial e imprimirle así un carácter más racional y científico a los procesos y actividades de innovar y desaprender. Entre estas tres capacidades debe haber una interacción y fortalecimiento permanente. Para los propósitos del Sistema de Conocimiento DICES no se concibe ninguna de ellas sin el concurso de las otras dos, ni mucho menos de manera aislada e independiente, forman un sistema [56], el Sistema de Capacidades DIA, denominado así no tanto porque coincide con el acrónimo conformado por las iniciales del nombre de cada capacidad, sino porque representa, como el día solar, la claridad, en este caso conceptual, indispensable para el proceso de transformación de los elementos del Sistema de Conocimiento DICES, cuya finalidad última es llegar al máximo estado: la sabiduría.

En consecuencia, la implantación de la gestión del conocimiento implica iniciar con la dotación de competencias laborales al personal de las pyme encadenando la adquisición de las 
capacidades: DIA, de asimilación de información estratégica, estratégica, tecnológica, de innovación y de actuación.

\section{REFERENCIAS BIBLIOGRÁFICAS}

[1] ME Porter (1990) La ventaja competitiva de las naciones, CECSA, Buenos Aires

[2] S Ghoshal et al (1999) "A new manifesto for management". Sloan Management Review. Spring,

[3] JI Dalmau (1999) Competencia y estrategia. Universidad Politécnica de Valencia

[4] J Garay et al (1997) La Industria de América Latina ante la globalización. Tomo I. Colombia: estructura industrial e internacionalización 1967-1996

[5] RF De Rato (2000) en La Pequeña y MeDIAna Empresa en España. Políticas y Realizaciones (1996-2000), Ministerio de Hacienda de España

[6] European Commission (1996) Action Plan for Innovation.

[7] A Toffler et al (1994) Las guerras del futuro. Plaza \& Janes. España.

[8] RM Kanter (2000) "La gestión en un mundo globalmente conectado". Harvard Deusto Business Review, Julio-Agosto

[9] C Bartlett, S Ghoshal (2000) "Going global: lessons from late movers". Harvard Business Review, March-April

[10] P Drucker (1998) La organización del futuro. Ediciones Deusto

[11] C Freeman (1999) The economics of industrial innovation. Londres: Francés Pinter

[12] P Drucker (2000) "La productividad del trabajador del conocimiento: máximo desafío". Harvard Deusto Business Review, Sep./Oct

[13] I Monaka, H Takeuchi (1990) "The knowledge creating company: How japanese companies create the dynamics of innovation" Harvard Business Review May-June

[14] Ackoff, R. (1993) Las fábulas antiburocráticas de Ackoff. Granica, Barcelona

[15] P Drucker (2000) "Más allá de la revolución de la información". Harvard Deusto Business Review, Ene./Feb

[16] D Marchand (2000) "Gestión y tecnología: un desafío para la alta dirección". Harvard Deusto Business Review, Mar./Abr

[17 ]Haour (1998) "Managing technical innovation as a global process". Perspectives for Managers $M^{\circ} 2$, IMD, February

[18] P Escorsa y J Valls (1997) Tecnología e innovación en la empresa. Dirección y gestión. Universidad Politécnica de Cataluña

[19]CK Prahalad y G Hamel (1990) "The core competences of the corporation". Harvard Business Review, may-june, Pp. 79-91

[20] JA Schumpeter (1934) The Theory of Economic Development, Cambridge, MA: Harvard University Press

[21] B Lundvall (1992) Mational Systems of Innovation. Compilado por Bengt-Ake Lundvall. Pinter Publishers. Londres

[22] SJ Kline y M Rosenberg (1986) An Overview of Innovation. The Mational Academy Press, Washington D.C

[23] I Fernández y F Conesa (1996). Estructuras de Interfaz en el Sistema Español de Innovación. Su Papel en la Difusión de Tecnología. CTT, Universidad Politécnica de Valencia

[24] M Porter (1985) "Technology and competitive advantage" Journal of Business Strategy V5 Mo 3.

[25] PF Drucker (1993) "Professionals' productivity", Across the Board, Vol. 30 Mo. 9, Mov/Dec,

[26] G Hamel (1999) "Innovación estratégica y creación de valor". Harvard Deusto Business Review, May./Jun

[27] B Muñoz-Seca (2000) "Towards a Europe of Knowledge: Policy spaces to generate learning". Paper presented in Lisbon Seminar Towards a Learning Society 28-29 May

[28] RT Pascale (2000) "Equilibrios al borde del caos". Harvard Deusto Business Review, Ene./Feb.

[29] M Wheatley (1994) El Liderazgo y la Mueva Ciencia. Granica, Argentina

[30] S Kauffman (1993). The Origins of Order: 
Self-Organization and Selection in Evolution. Oxford University Press. Mew York.

[31] S Kauffman (1995) At Home in the Universe. Viking.London

[32] Holland (1995) Hidden Order. Reading: Addison-Wesley

[33] RM Grant (1996) Dirección Estratégica. Editorial Civitas

[34] J Barney (1986) "Organizational culture: Can it be a source of sustained competitive advantage?", Academy of Management Review, 11, pp. 656-665

[35] P Adler (Ed.) (1992) Technology and the future of work, Oxford Univ. Press, Oxford

[36] J Bessant (1989) "Microelectronics and change at work", I.L.O., Ginebra

[37] M Novick y MA Gallart (1997) Competitividad, redes productivas y Competencias Laborales, CIMTERFOR OIT. Montevideo

[38] JB Kassarjian (1998) "La paradoja de dirigir un cambio". Harvard Deusto Business Review, Sep./Oct

[39] C Bartlett C y S Ghoshal (1995) "Changing the Role of Top Management: Beyond Systems to People", Harvard Business Review, May - June

[40] E De Miguel (1993) Introducción a la Gestión. Universidad Politécnica de Valencia

[41] P Drucker (1992) "The new society of organizations". Harvard Business Review, september

[42] PM Senge (1992) La quinta disciplina. Granica, Barcelona

[43] I Monaka, H Takeuchi (1990) "The knowledge creating company: How japanese companies create the dynamics of innovation" Harvard Business Review, May-June.

[44] H Mintzberg (1994) The rise and fall of strategic planning. Free Press, Mew York

[45] CK Prahalad, G Hamel (1990) "The core competences of the corporation", Harvard Business Review, may-june, Pp 79-91

[46] Bengt-Åke Lundvall (2000) "Europe and the learning economy on the need for reintegrating the strategies of firms, social partners and policy makers". Lisboa Seminar, May 28-30, 2000.

[47] ME Porter (2000) En Hodgetts, R.M. "Conversación con Michael E. Porter: la estrategia y la estructura organizativa". Harvard Deusto Business Review. May/Jun.

[48] K Ishikawa (1991). Qué es el Control Total de Calidad. Bogotá, Morma. 1991

[49] R Ackoff (1993) Las fábulas antiburocráticas de Ackoff. Granica, Barcelona

[50] PF Drucker (1994) La sociedad postcapitalista. Editorial Morma, Bogotá

[51] C Matus (1993) Sobre la teoría de las Macroorganizaciones. Fundación Altadir

[52] M Van Der Erve (1990) El Futuro de la Gerencia. Visión y cultura corporativa Legis, Bogotá

[53] C Argyris y DA Schon (1996) Orgazniational learning II: Theory, method and practice. Addison Wesley, Reading, MA

[54] P Senge (1999) A conversation with Peter Senge: New Developments in Organizational Learning. Organizational Dynamics

[55] H Steedman (2000) "New skill needs and competence building to promote social cohesion." Paper presented in Lisbon Seminar Towards a Learning Society 28-29 May 